\title{
Construction of a matrix assisted laser desorption/ ionization time-of-flight mass spectrometer
}

\author{
R.V. Coorey ${ }^{*}$, T.R. Ariyaratne, D.D.N. Barlo Daya and \\ W.M.K.P. Wijayaratna
}

Department of Physics, University of Colombo, Colombo 03, Sri Lanka

\begin{abstract}
A matrix-assisted laser desorption/ionization time-of-flight (MALDI-TOF) mass spectrometer has been constructed at the Department of Physics, University of Colombo, Sri Lanka. In this mass spectrometer, a solid sample which contains analyte molecules embedded in a suitable matrix compound in excess is exposed to a UV pulsed nitrogen laser source $(\lambda=337 \mathrm{~nm})$. The desorbed secondary ions from the sample are mass analyzed using the time-of-flight technique. The first MALDI-TOF mass spectra of two peptides namely [tyr ${ }^{8}$ ]-bradykinin $(1076.2 \mathrm{u})$ and porcine $\mathrm{N}$-acetyl renin substrate tetradecapeptide (1801.1 u) with the matrix 2,5 di-hydro benzoic acid $(154.1 \mathrm{u})$ have been obtained. Each peptide produced a $\mathrm{MH}^{+}$peak corresponding to its intact molecular ion with $\mathrm{m} / \mathrm{q}$ values of $1089 \pm 6$ and $1794 \pm 8$ respectively.
\end{abstract}

\section{INTRODUCTION}

Sir J.J. Thomson's measurement of charge to mass $(\mathrm{q} / \mathrm{m})$ ratio of the electron in 1897 has given birth to mass spectrometry. Since then, mass spectrometry has been used mainly for mass and structural determination of molecules below $1 \mathrm{kDa}$. A new era in mass spectrometry has dawned only after the introduction of plasma desorption mass spectrometry (PDMS) ${ }^{1}$ by Macfarlane \& co-workers at the Texas A \& M University, USA in 1974. PD-MS was the only technique that could be used at that time for the mass analysis of large bio-molecules that are nonvolatile and thermally labile. Those molecules could not be mass analyzed using conventional mass spectrometric techniques such as electron impact ionization (EII) technique and later the chemical ionization (CI).

\footnotetext{
${ }^{*}$ Corresponding author
} 
PDMS has been applied successfully in the mass analysis of bio molecules up to 45,000 u. $^{2}$

In the early 1960's lasers were used to generate ions in mass spectrometers ${ }^{3}$ and the first systematic attempts to produce ions of organic molecules were made in the early 1970 's ${ }^{4}$. Since then, several experiments were performed with laser desorption ionization mass spectrometry (LDI-MS). Conzenmius and Capellen have published complete reviews of the applications of LDI-MS with extensive references ${ }^{5}$. In 1987, Karas and Hillenkamp showed that by using a low concentration of analyte molecules embedded in a relatively larger amount of suitable matrix of low molecular weight, it was possible to desorb and ionize very large bio-molecules ${ }^{6}$. This technique was called matrix-assisted laser desorption/ionization mass spectrometry (MALDI-MS). In this technique, the matrix absorbs most of the laser energy and the resulting energisation leads to a phase transfer, ionization and formation of stable molecular ions ${ }^{7}$. Using MALDI-MS, it is possible to detect intact molecules having molecular weights greater than 200,000 $\mathrm{u}^{8}$.

The construction of a MALDI-TOF mass spectrometer at the Department of Physics, University of Colombo was deemed necessary for several reasons. The capability of the MALDI technique to detect large bio molecules has been well established. Thus it would be a useful analytical tool for local bio-chemists. The viability of the MALDI technique to handle low mass molecules has been demonstrated ${ }^{9}$. Thus it would facilitate the study of low mass organic molecules. In addition, the higher sensitivity that the MALDI technique has over the PD technique in detecting molecular ions in crude samples ${ }^{10}$ and in mixtures ${ }^{11}$ is considered as a distinct advantage in local natural product analysis.

\section{EXPERIMENTAL}

\subsection{Details of the Construction}

Fig.1 shows the schematic diagram of the MALDI-TOF mass spectrometer. It basically consists of a laser ion source, optical components and a time-of-flight mass analyzer. The ion source contains a pulsed UV nitrogen laser (VSL-337ND from Laser Science Inc., USA) having a pulse duration 3 ns (FWHM) and maximum output energy $\sim 250 \mu \mathrm{J}$. The laser ion source is coupled to a time-of-flight mass analyser via several optical components. The time-of-flight mass analyser is mounted in a vacuum chamber. The construction details of the vacuum chamber is described elsewhere ${ }^{12}$. The vacuum chamber and its feed through ports are used to house the target holder and the acceleration grid. A stainless steel tube is used to provide the necessary flight path for 
the desorbed ions. A DC $5 \mathrm{~V}$ power supply was used to trigger the laser source manually using a TTL $5 \mathrm{~V}$ pulse.

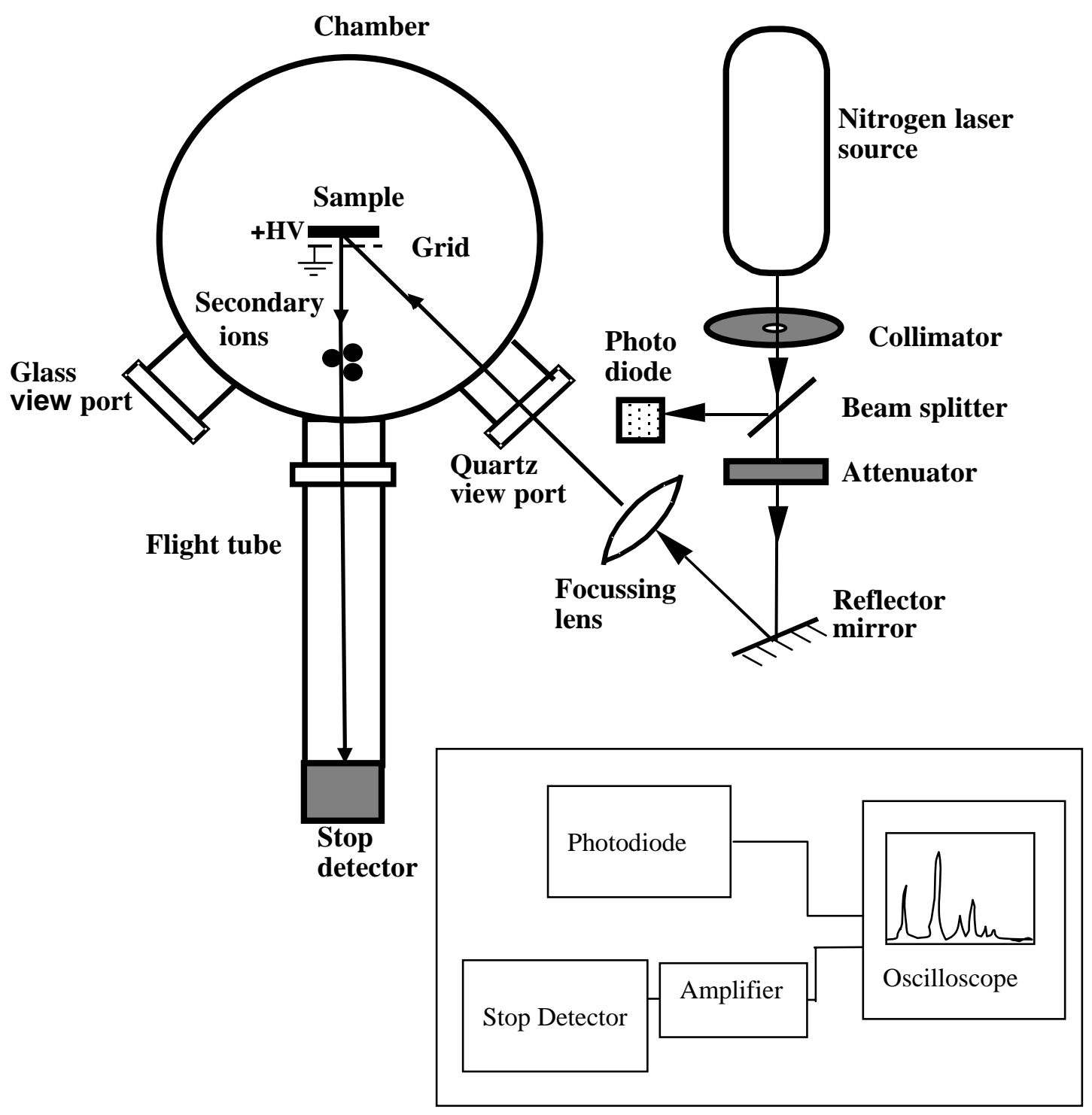

Fig. 1 Schematic diagram of the matrix-assisted laser desorption ionization time-of-flight mass spectrometer (MALDI-TOF-MS). $\mathrm{N}_{2}$ laser is used to send pulses on to a sample. Desorbed positive ions are detected by an MCP stop detector. Inset is the data acquisition system.

In order to generate a start trigger pulse for the time-of-flight measurement, the pulsed laser beam was allowed to pass through a beam splitter and the deviated component to hit a photodiode. The output pulse from the photodiode was then used to trigger a $300 \mathrm{MHz}$ digital storage oscilloscope (Lecroy 9310 Dual, Lecroy Corporation, 
USA) having a sampling rate of 100 mega samples per second. The start trigger pulse, as measured by the digital storage oscilloscope, typically has an amplitude of 3 to 4 volts, with a pulse width (FWHM) of about 25 ns and a rise time of about 8 ns. The stop detector is made of two micro channel plates (MCP) coupled in tandem. It provides the stop signal for the time-of-flight measurement.

As shown in fig. 1, the UV laser was used to send pulses through the quartz view port onto the target, a solid sample maintained at a high voltage of $+10 \mathrm{kV}$, with $45^{\circ}$ angle of incidence. The pulsed laser beam was initially collimated to give a circular spot of approximately $5 \mathrm{~mm}$ diameter. The incident laser beam was further focussed into a tiny spot of diameter less than $0.5 \mathrm{~mm}$ on the target by means of a quartz lens. An optical mirror was used to direct the laser beam onto the target and scan over the sample as necessary. An ordinary glass plate (thickness $\sim 6.0 \mathrm{~mm}$ ) mounted on one of the ports was used to observe the position of the laser spot on the target. The intensity of the laser pulses were controlled by using a variable attenuator (LASEROPTIK, Germany) and kept above a threshold value to optimize the ion production. After accelerating the desorbed positive ions from the sample through a distance of $4.0 \mathrm{~mm}$, they are allowed to drift through a field free region and are finally detected by the MCP stop detector mentioned earlier. A post acceleration voltage over a distance of $5.0 \mathrm{~mm}$, just before the stop detector, was introduced in order to enhance the ion detection. This MCP detector output was used to stop the time counting. The time difference between the start pulse and a stop pulse is a measure of the time-of-flight of the desorbed ion concerned. In order to record the time-of-flight, the start signal and stop signals (amplified by a fast timing amplifier, ORTEC 574) were fed to the digital oscilloscope (inset in fig. 1). Initially, the time-of-flight spectra were saved as Lecroy files and were re-produced later and printed using MS Excel 97 soft-ware package. The vacuum of the MALDI-TOF mass spectrometer was maintained around $10^{-6}$ mbar using an oil diffusion pump backed by a rotary pump.

\subsection{Sample Preparation}

The analytes, tyr $^{8}$ ]-bradykinin $(1076.2 \mathrm{u})$ and porcine N-acetyl-renin substrate tetradecapeptide (1801.1 u), and the matrix 2,5 di-hydro benzoic acid (DHB) (154.1 u) were obtained from Sigma chemicals, St. Louis, USA and used without further purification.

All the samples used for analysis by MALDI were prepared as follows. The matrix DHB was dissolved in a mixture of ethanol and distilled water, 1:9 v/v (conc. 10 $\mathrm{g} / \mathrm{l}$ ). Each analyte used was dissolved in distilled water (conc. $10^{-5} \mathrm{M}$ ). Approximately, $1 \mu \mathrm{l}$ of matrix and $1 \mu \mathrm{l}$ of each analyte was deposited on an aluminium disc, dried in an exhaust, and used for the analysis. 


\section{RESULTS AND DISCUSSION}

The laser trigger signal peak was observed in all MALDI mass spectra. The time-of-flight of a desorbed ion was determined by using the time difference between the centroid time of the laser trigger signal peak (taken as the reference) and the centroid time of an ion peak corresponding to the desorbed ion. MALDI-TOF spectra were taken by setting a $2 \mu$ s delay in the oscilloscope in order to check for any pre-laser events. Prelaser events may arise from background signals due to stray molecular fragments or electrons emitted from the sample, noise generated in the stop detector etc., which could occur before the laser is fired. However, no such events have been observed.

It can be shown that the experimental time-of-flight $(t)$ of a desorbed ion is given by $^{13}$,

$t=\sqrt{\frac{2 m}{q}}\left[\frac{S_{1}\left(\sqrt{V_{1}+V_{2}}-\sqrt{V_{0}}\right)}{V_{1}}+\frac{S_{2}}{2 \sqrt{V_{1}+V_{0}}}+\frac{S_{3}\left(\sqrt{V_{2}+V_{1}+V_{0}}-\sqrt{V_{1}+V_{0}}\right)}{V_{2}}\right]+T_{0}$,

where $m$ - mass of the ion,

$q$ - charge of the ion,

$S_{1}$ - accelerating distance,

$S_{2}$ - field free distance,

$S_{3}$ - post accelerating distance,

$V_{0}$ - voltage corresponding to the initial average kinetic energy of the ion,

$V_{1}$ - accelerating voltage,

$V_{2}$ - post accelerating voltage.

$T_{0}$ - mass spectrometer constant

For the mass spectrometer, the following parameters were measured:

$S_{1}=4.0 \mathrm{~mm}$

$S_{2}=28.2 \mathrm{~cm}$

$S_{3}=5.0 \mathrm{~mm}$

The following values were set:

$V_{1}=+10 \mathrm{kV}$

$V_{2}=-2.5 \mathrm{kV}$

Initially, 3 DHB matrix ion peaks were identified by determining their masses using the observed times-of-flight values (directly read from the mass spectra) and using a simplified time-of-flight equation. The DHB matrix (154.1 u) has shown a few characteristic peaks corresponding to $\mathrm{MH}^{+}$and $[\mathrm{M}-\mathrm{OH}]^{+}$ions. A peak corresponding to 
$[\mathrm{M}+\mathrm{Al}]^{+}$ion was also detected in the MALDI spectrum of the DHB matrix. This could originate from the aluminium sample backings. (Table 1).

The initial energies of the secondary ions were considered using the fact that the desorbed ions in MALDI-TOF-MS in the mass range of $1000 \mathrm{u}-15,600 \mathrm{u}$ have an average velocity of approximately $750 \mathrm{~ms}^{-1}{ }^{14}$. The time-of-flight equation shown above was then used to evaluate $T_{0}$ for the 3 matrix ions. The average value of $T_{0}$ was found to be 16 ns. The $T_{0}$ is the time correction of the theoretical time-of-flight of an ion and is considered to be a mass spectrometer constant. The experimental time delays that could occur in both the start and stop events contribute to the $T_{0}$ value. In the start event there could be a time lag between the desorption/ionisation event and the recording of the start trigger pulse by the digital oscilloscope. In the stop event there could be a time lag between the generating and the recording of the stop pulse by the MCPs and the digital oscilloscope respectively. The time jitter associated with the laser diode and propagation delays that occur in the amplifier may contribute to the time lag mentioned above. The calculated value of $T_{0}$ was found to agree with the estimated total propagation time delays.

Table 1: Time-of-flights (TOF), full width at half maximum (FWHM) and initial energies of observed ions are shown. The calculated $m / q$ value of the $\mathrm{MH}^{+}$ion of each analyte is given. The theoretical $\mathrm{m} / \mathrm{q}$ values of analyte and matrix ions are also provided.

\begin{tabular}{|l|c|c|c|c|c|}
\hline & $\begin{array}{c}\text { Observed } \\
\text { TOF } \\
t_{o b}(\mathrm{~ns})\end{array}$ & $\begin{array}{c}\text { FWHM } \\
\text { (ns) }\end{array}$ & $\begin{array}{c}\text { Initial } \\
\text { energy } \\
(\mathrm{eV})\end{array}$ & $\begin{array}{c}\text { Calculated } \\
\mathrm{m} / \mathrm{q}\end{array}$ & $\begin{array}{c}\text { Theoretical } \\
\mathrm{m} / \mathrm{q}\end{array}$ \\
\hline DHB matrix & 2590 & 20 & 0.45 & - & 155.1 \\
$\mathrm{MH}^{+}$ & 2510 & 20 & 0.40 & - & 137.1 \\
{$[\mathrm{M}-\mathrm{OH}]^{+}$} & 2820 & 20 & 0.53 & - & 181.1 \\
{$[\mathrm{M}+\mathrm{Al}]^{+}$} & & & & & \\
\hline [Tyr $\left.^{8}\right]_{-}$ & & 20 & 3.14 & 1088.8 & 1077.2 \\
bradykinin $_{\mathrm{MH}^{+}}$ & 6970 & & & & \\
\hline $\begin{array}{l}\text { N-acetyl-renin } \\
\text { substrate tetra- } \\
\text { decapeptide }\end{array}$ & & & & & \\
$\mathrm{MH}^{+}$ & 8960 & 20 & 5.25 & 1794.4 & 1802.1 \\
\hline
\end{tabular}

Figs. 2 to 4 show the first single shot positive ion MALDI mass spectra obtained from the newly built MALDI mass spectrometer with the samples of $\operatorname{tyr}^{8}$-bradykinin and $\mathrm{N}$-acetyl renin substrate tetradecapeptide and that of a mixture of $\operatorname{tyr}^{8}$-bradykinin and $\mathrm{N}$ - 
acetyl renin substrate. The average value of $T_{0}$ and other known parameter values were used to calculate the masses of the $\mathrm{MH}^{+}$ion peak of each analyte. (Table 1). The $\mathrm{m} / \mathrm{q}$ values for the $\mathrm{MH}^{+}$ion peak of $\left[\mathrm{tyr}^{8}\right]$-bradykinin and $\mathrm{N}$-acetyl renin substrate tetradecapeptide were estimated to be $1089 \pm 6$ and $1794 \pm 8$ respectively. The main reason for the shift in the experimental $\mathrm{m} / \mathrm{z}$ values from the theoretical values is the uncertainty in the measurement of the observed time-of-flight values of the ions. The quite large peak width (FWHM of 25 ns) of the laser trigger signal and the time jitter associated with the laser diode contribute to the uncertainty of the observed time-offlight values of the ions.

The storage oscilloscope has only a sampling rate of 100 mega samples per second (i.e. 100 samples per $\mu$ s) and it corresponds to a time uncertainty of 10 ns. Thus the experimentally measured mass peaks were restricted by the time resolution of the digital oscilloscope. Furthermore, the mass resolution of this MALDI mass spectrometer was also restricted by the relatively short fight length $(29.1 \mathrm{~cm})$ used in these measurements. In addition, the deviation of the $S_{1}, S_{2}$ and $S_{3}$ values from their corresponding geometrical values could contribute to their uncertainty.

The mass resolution achievable was only around 200 for the analyte ions. However, in these preliminary measurements, the newly built MALDI-TOF-MS has been applied successfully to obtain the first MALDI spectra and to detect molecules in the mass range 100 - $2000 \mathrm{u}$. For future work, plans are being made to build a delayed extraction MALDI (DE-MALDI) system. The principle of DE-MALDI is based on an idea by Wiley and McLaren who have theoretically shown that by using a two-field pulsed ion source, it is possible to provide a first order correction for the initial space distribution of the ions ${ }^{15}$. In the DE-MALDI technique, the ions are initially produced in a field-free region and subsequently an accelerating field is turned on by the application of a fast pulse with a pre-determined delay time after the initial ion formation. The DEMALDI technique with the addition of an electrostatic ion reflector has proved to be a great success in improving the mass resolution of a MALDI-TOF mass spectrometer ${ }^{16}$.

\section{CONCLUSIONS}

A matrix assisted laser desorption/ionization mass spectrometer was designed, constructed and tested at the Department of Physics, University of Colombo, Sri Lanka, using a UV pulsed nitrogen laser having a wavelength of $337 \mathrm{~nm}$. The first mass spectra from the newly built MALDI mass spectrometer were collected successfully using [tyr ${ }^{8}$ ]bradykinin (1076.2 u) and porcine N-acetyl-renin substrate tetradecapetide (1801.1 u) as compounds with the DHB matrix. 


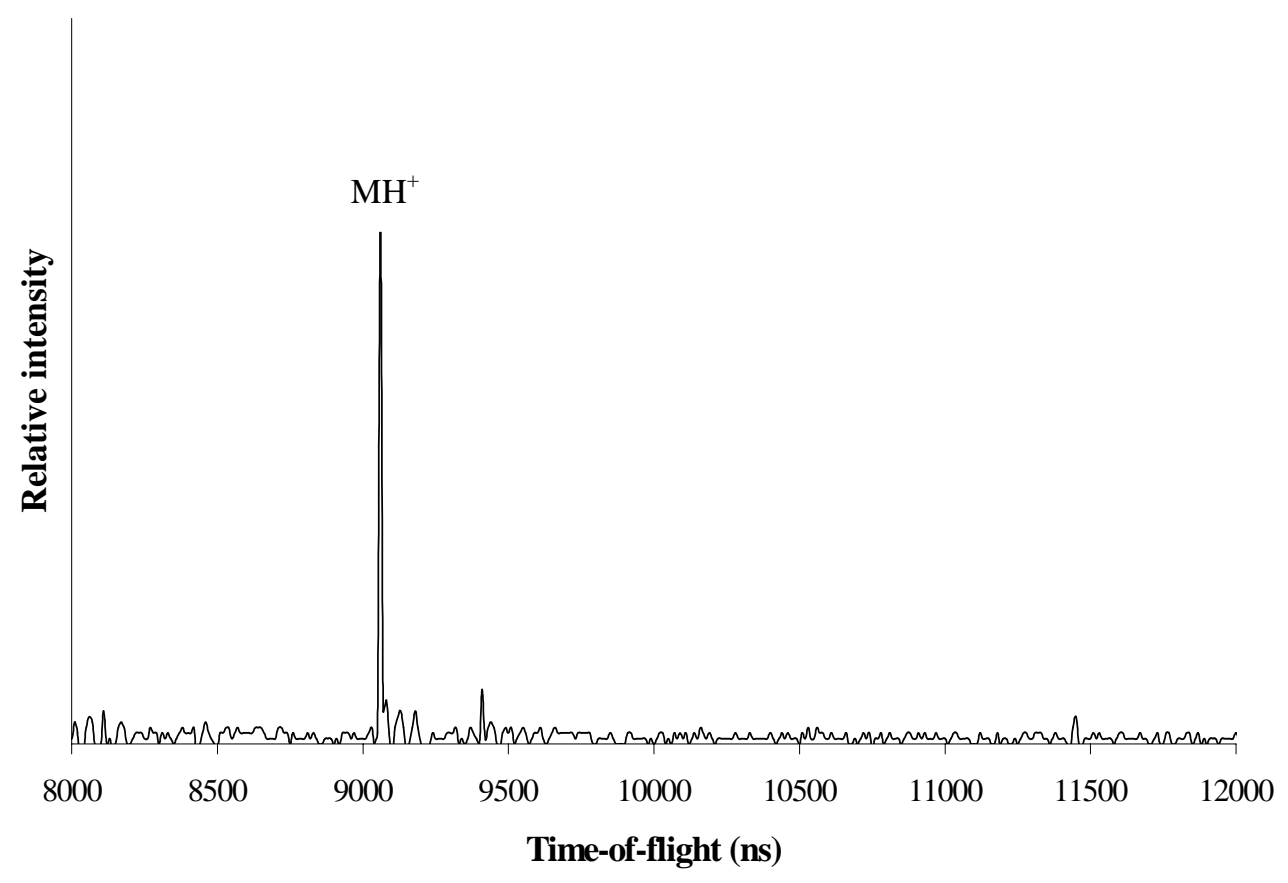

Fig. 2: Single shot positive ion MALDI spectrum of $\left[\operatorname{tyr}^{8}\right]$-bradykinin

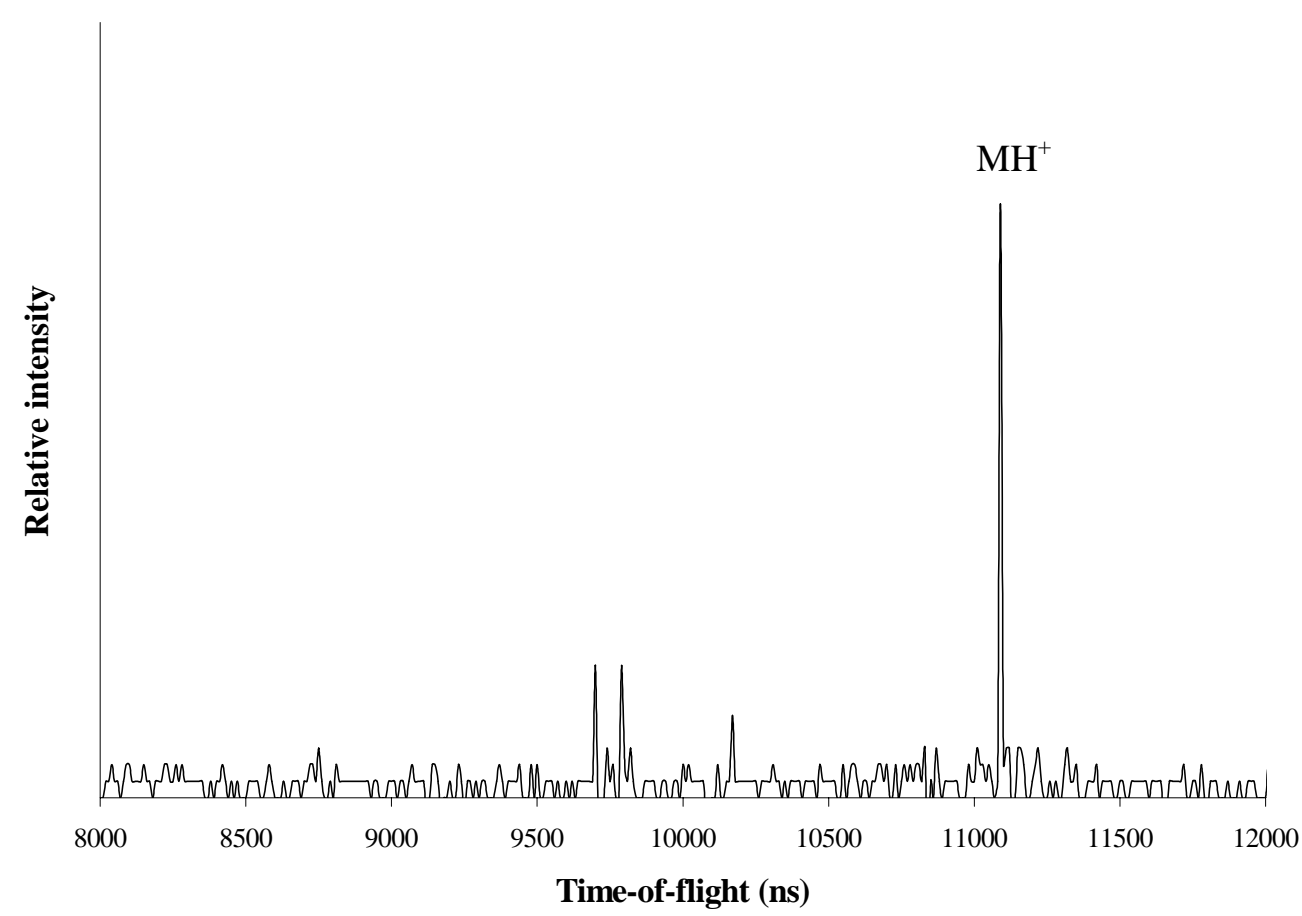

Fig.3: Single shot positive ion MALDI spectrum of $\mathrm{N}$-acetyl renin substrate tetradecapeptide 


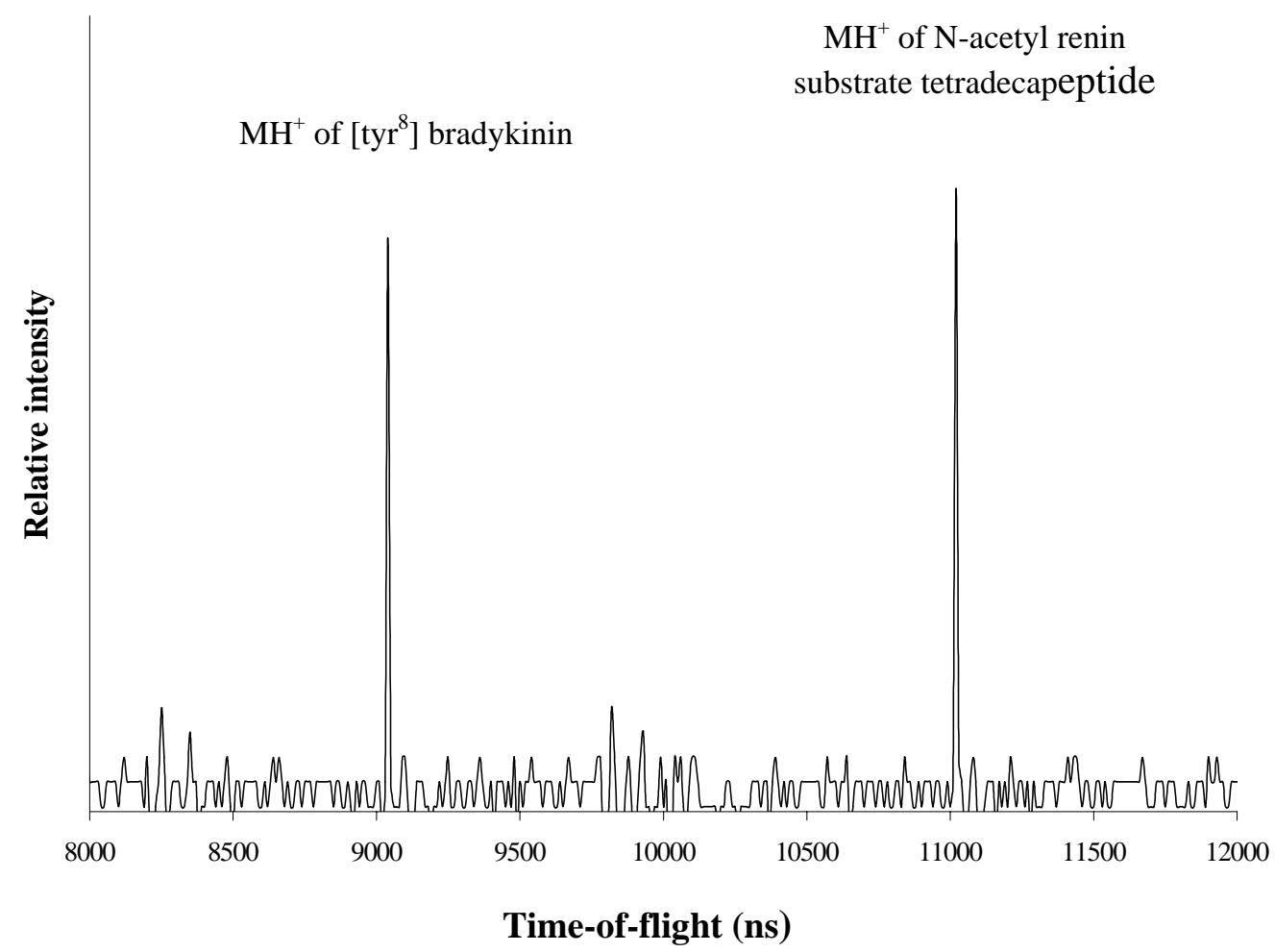

Fig. 4 Single shot positive ion MALDI spectrum of a mixture of [tyr $\left.{ }^{8}\right]$-bradykinin \& N-acetyl renin substrate tetradecapeptide

\section{ACKNOWLEDGEMENTS}

The financial assistance provided by the Third World Academy of Sciences (TWAS) and the International Programs in the Physical Sciences (IPPS) of the Uppsala University, Sweden and the donations of some necessary instruments by the Japan International Corporation Agency (JICA) are gratefully acknowledged. The technical assistance received from the mechanical and electronic workshops of the Department of Physics, University of Colombo is also acknowledged.

\section{REFERENCES}

1. D.F. Torgerson, R.P. Skowronski and R.D. Macfarlane, New approach to the mass spectrometry of non-volatile compounds, Bio-chem.Biophy.Res. Commun. $\underline{60}, 616$ (1974).

2. G. Jonsson et.al., Rapid Commun. Mass Spectrom.3, 190 (1989). 
3. F.J. Vastola, O. Mummar and A.J. Pirne, Org. Mass Spectrum, 3, 101 (1970).

4. M.A. Posthumus, P.G. Kistemaker, H.L.C. Meuzelaar and M.C. Ten Neuver de Brauw, Laser desorption mass spectrometry of polar nonvolatile bio-organic molecules, Anal Chem., $\underline{50}, 985$ (1978).

5. R.J. Conzemius and J.M. Capellen, A review of the applications to solids of laser ion source in mass spectrometry, Int.J.Mass Spectrom.Ion Phys. 34, 197 (1980).

6. M. Karas, D. Bachmann, U. Bahr and F. Hillenkamp, Matrix-assisted ultra violet laser desorption of non-volatile compounds, Int. J. Mass Spectrum. Ion proc, $\underline{78}$, 53 (1987).

7. K.L. Busch, Desorption ionization mass spectrometry, Journal of mass spec, $\underline{30}$, 233 (1995).

8. F. Hillenkamp, Ultraviolet laser desorption/ionization of biomolecules in the high mass range, Proceedings of the $37^{\text {th }}$ ASMS Conference on Mass Spectrometry and allied topics (Florida, 1989) p.1168.

9. R. Lidgard, M.K. Duncan, Utility of matrix-assisted laser desorption/ ionization time of flight mass spectrometry of low molecular weight compounds, Rapid Commun. Mass Spectrom., $\underline{9}, 128$ (1995).

10. Z. Dinya et.al., Studies of the components of crude desertomycin complex bymeans of electrospray and matrix-assisted laser desorption/ionization mass spectrometric techniques, Rapid Commun. Mass Spectrom., 10, 1439 (1996).

11. R.C. Beavis and B.T. Chait, Rapid sensitive analysis of protein mixtures by mass spectrometry, Proc. Natl. Acad. Sci., USA, 87, p 6873 (1990).

12 C.K.G. Piyadasa, Performance of the newly constructed linear time-of-flight plasma desorption mass spectrometer, Proceedings of the $51^{\text {st }}$ Annual Session, Sri Lanka Association for the Advancement of Science E1-06 (1995) p. 341.

13. S. Widdiyasekera, Initial velocity distribution of secondary ions in electronic sputtering of organic molecules, Ph.D. thesis, Uppsala University, Sweden, 1987

14. R.C. Beavis and B.T. Chait, Velocity distributions of intact high mass polypeptide molecule ions produced by matrix assisted laser desorption., Chem.Phys. letters, 181, 479 (1991).

15. W.C. Wiley and I.H. McLaren, Time-of-flight mass spectrometer with improved resolution, Rev.Sci.Instrum., 26, 1150 (1953)

16. M.L. Vestal, P. Juhasz and S.A. Martin, Delayed extraction matrix-assisted laser desorption time-offlight mass spectrometry, Rapid Commun.Mass Spectrom., 9, 1044 (1995). 